\title{
The contact process seen from a typical infected site
}

\author{
Jan M. Swart \\ Institute of Information Theory and Automation of the ASCR (ÚTIA) \\ Pod vodárenskou věží 4, 18208 Praha 8, Czech Republic \\ swart@utia.cas.cz
}

September 3, 2008

Abstract

We consider contact processes on general Cayley graphs. It is shown that any such contact process has a well-defined exponential growth rate, which can be related to the configuration seen from a 'typical' infected site at a 'typical' late time. Using this quantity, it is proved that on any nonamenable Cayley graph, the critical contact process dies out.

\section{Cayley graphs}

Let $\Lambda$ be a countable, finitely generated group, with group action denoted by $(i, j) \mapsto i j$, inverse operation $i \mapsto i^{-1}$, and unit element (origin) 0 . Let $\Delta \subset \Lambda$ be a finite generating set for $\Lambda$ that is symmetric in the sense that $i \in \Delta$ implies $i^{-1} \in \Delta$. Then the (left) Cayley graph $\mathcal{G}(\Lambda, \Delta)$ associated with $\Lambda$ and $\Delta$ is the graph with vertex set $\Lambda$, where there is an edge connecting $i, j \in \Lambda$ if and only if there is a $k \in \Delta$ such that $j=k i$. Examples of Cayley graphs are $\mathbb{Z}^{d}$, equipped with the usual nearest-neighbor structure, or the regular tree $\mathbb{T}_{d}$ in which each site has $d+1$ neighbors
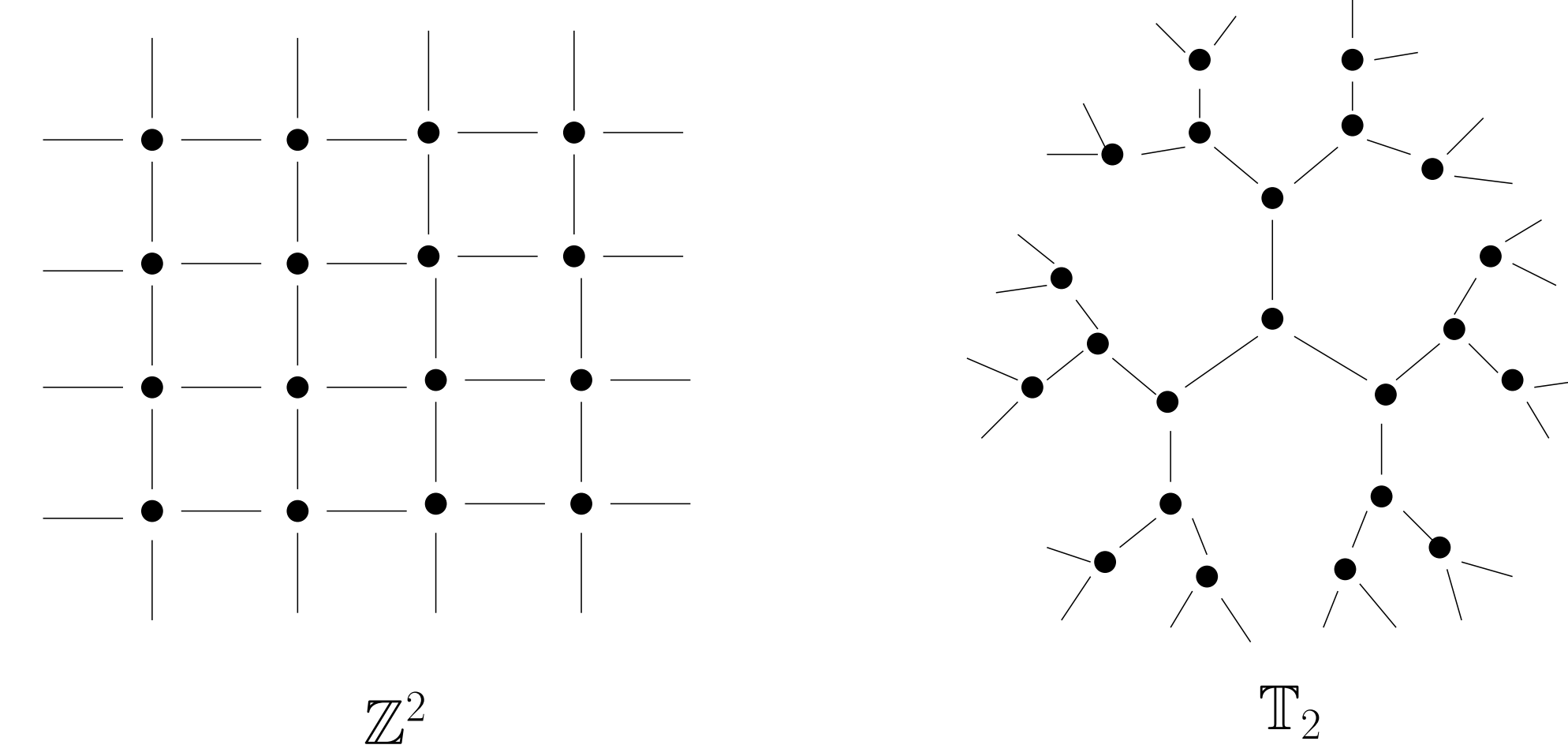

\section{Amenability and exponential growth}

A Cayley graph $\mathcal{G}(\Lambda, \Delta)$ is called amenable if for every $\varepsilon>0$ there exists a finite nonzero $A \subset \Lambda$ such that

$$
\frac{|\partial A|}{|A|} \leq \varepsilon \quad \text { where } \quad \partial A:=\{i \notin A: \exists \operatorname{edge}(i, j) \text { s.t. } j \in A\} .
$$

This says that there exist large 'blocks' $A$ whose surface can be made arbitrarily small compared to their volume. For example, $\mathbb{Z}^{d}$ is amenable but $\mathbb{T}_{d}(d \geq 2)$ is not.

A subadditivity argument shows that for each Cayley graph $\mathcal{G}(\Lambda, \Delta)$, the limit

$$
\lim _{n \rightarrow \infty} \frac{1}{n} \log |\{i \in \Lambda: d(0, i) \leq n\}|
$$

exists, where $d(0, i)$ denotes the usual graph distance of a site $i$ to the origin. The Cayley graph $\mathcal{G}(\Lambda, \Delta)$ is said to have exponential growth (resp. subexponential growth) if this limit is positive (resp. zero). It can be shown that subexponential growth implies amenability, but the converse is not true. A counterexample is the lamplighter group.

\section{Percolation}

In (nearest-neighbor, Bernoulli) percolation on a Cayley graph $\mathcal{G}(\Lambda, \Delta)$ we independently make edges open with probability $p$ and closed with the remaining probability. We let

$$
\theta(p):=\mathbb{P}[0 \leftrightarrow \infty]
$$

denote the probability that the origin is part of an infinite open cluster.

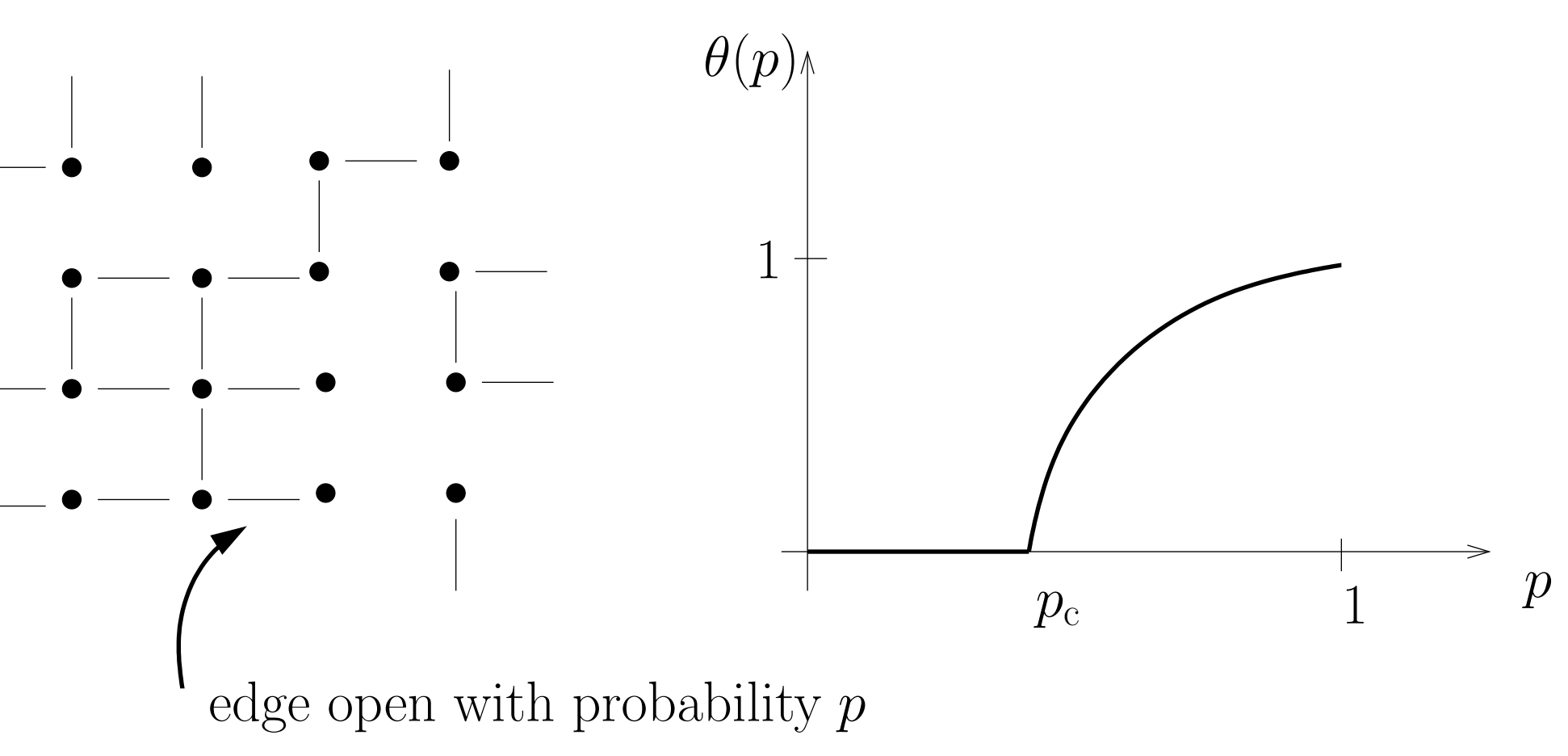

The graph of $\theta(p)$ is believed to be roughly as drawn above. In particular, there exists a critical percolation parameter $0<p_{\mathrm{c}}<1$ such that $\theta(p)=0$ for $p<p_{\mathrm{c}}$ and $\theta(p)>0$ for $p>p_{\mathrm{c}}$. On $\mathbb{Z}^{d}$, it is known that $\theta\left(p_{\mathrm{c}}\right)=0$ in dimension $d=2$ and dimensions $d \geq 1$. Proving this for $3 \leq d \leq 18$ is an open problem. For nonamenable graphs, the problem turns out to be easier than on $\mathbb{Z}^{d}$. In 1999, Benjamini, Lvons, Peres, and Schramm [BLPS99] proved that $\theta\left(p_{\mathrm{c}}\right)=0$ on any nonamenable Cayley graph.

\section{Contact processes}

The contact process with infection rate $\lambda$ on a Cayley graph $\mathcal{G}(\Lambda, \Delta)$ is a Markov process $\left(\eta_{t}\right)_{t>0}$ taking values in the subsets of $\Lambda$. If $i \in \eta_{t}$ then we say that the site $i$ is infected at time $t \geq 0$; otherwise we say that the site is healthy. Infected sites infect healthy neighboring sites with rate $\lambda$ and infected sites become healthy with recovery rate 1 .

The contact process $\left(\eta_{t}^{A}\right)_{t \geq 0}$ started from the initial state $A$ can be constructed with the help of a graphical representation. Here, a site $i$ is infected at time $t$ if there is a site $j \in A$ and an upward path from $(j, 0)$ to $(i, t)$ that may follow infection arrows but must avoid recovery symbols.

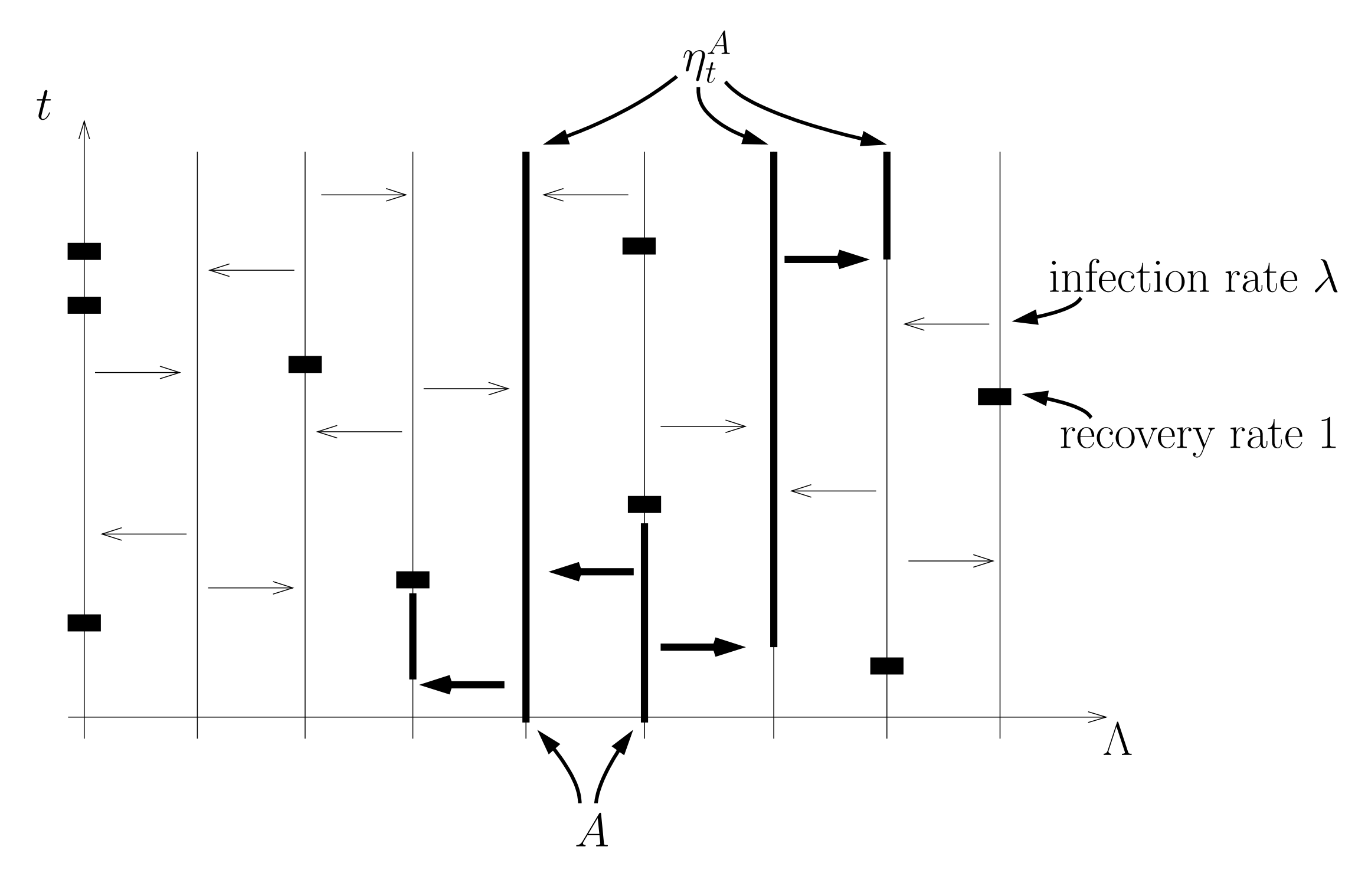

We let

$$
\begin{aligned}
\theta(\lambda) & :=\mathbb{P}\left[\eta_{t}^{\{0\}} \neq \emptyset \forall t \geq 0\right] \\
& =\mathbb{P}[(0,0) \rightarrow \infty]
\end{aligned}
$$

denote the probability that the process started with one infected site survives. The graph of $\theta(\lambda)$ is believed to be roughly as follows:

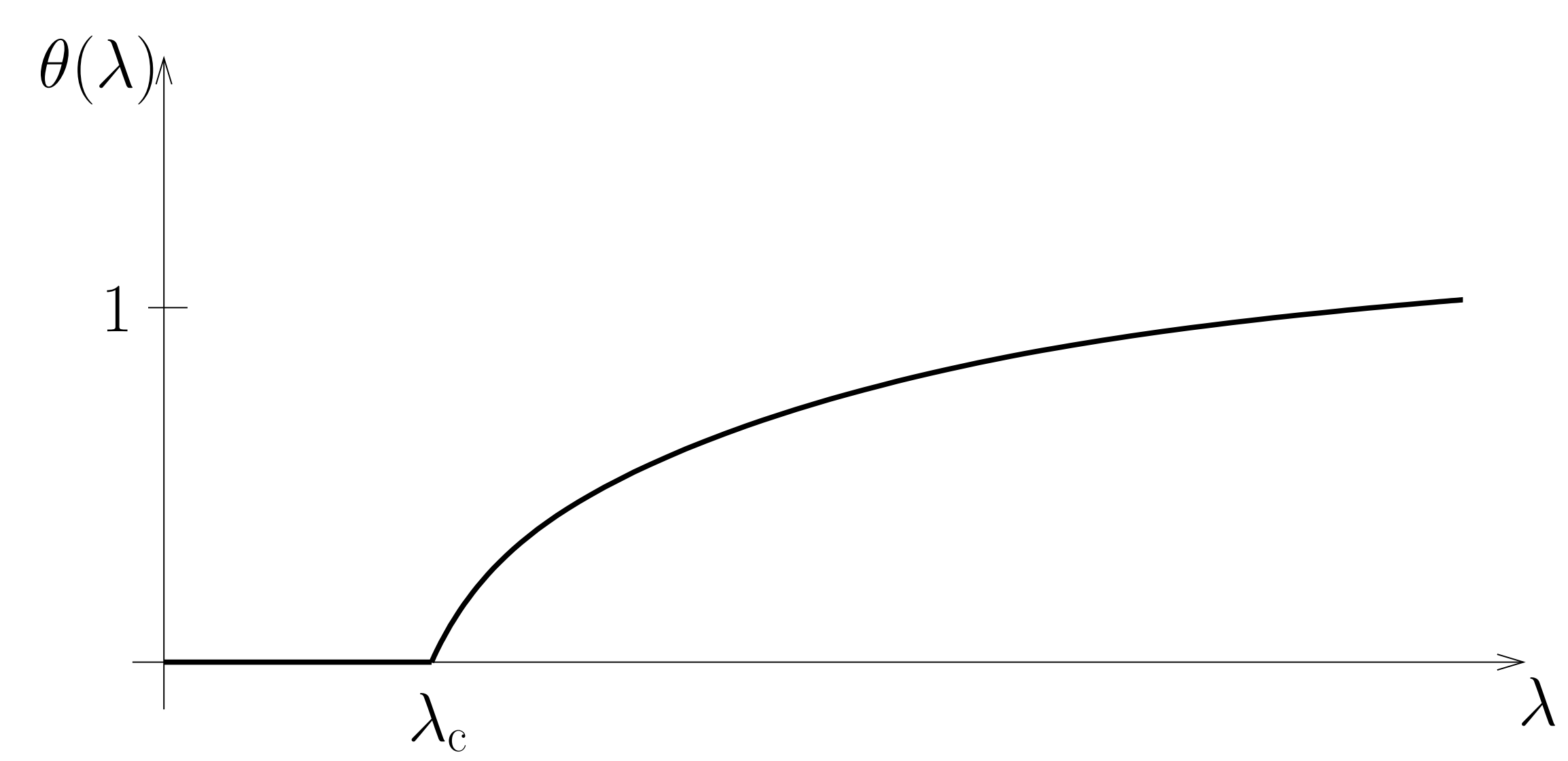

In particular, there exists a critical infection rate $0<\lambda_{\mathrm{c}}<\infty$ such that $\theta(\lambda)=0$ for $\lambda<\lambda_{\mathrm{c}}$ and $\theta(\lambda)>0$ for $\lambda>\lambda_{\mathrm{c}}$. In the celebrated paper of Bezuidenhout and Grimmett [BG90], it is proved that $\theta\left(\lambda_{\mathrm{c}}\right)=0$ for the process on $\mathbb{Z}^{d}$ in all dimensions $d \geq 1$. The analogue result for trees has been proved by Morrow, Schinazi, and Zhang in [MSZ94]. The next result generalizes this, in the spirit of [BLPS99], to any nonamenable Cayley graph:

Theorem [Swa08] Assume that $\Lambda$ is nonamenable.
Then $\theta\left(\lambda_{c}\right)=0$.

The exponential growth rate

A simple argument using subadditivity shows that each contact process on a Cayley graph has a well-defined exponential growth rate. More precisely, there exists a real constant $r=r(\lambda)$ such that the process started in any finite nonzero initial state satisfies:

$$
\lim _{t \rightarrow \infty} \frac{1}{t} \log \mathbb{E}\left[\left|\eta_{t}\right|\right]=r .
$$

If the Cayley graph has subexponential growth, then it is not hard to show that $r \leq 0$. In particular, on $\mathbb{Z}^{d}$, it is known that $r(\lambda)<0$ for $\lambda<\lambda_{c}$ and $r(\lambda)=0$ for $\lambda \geq \lambda_{c}$ [BG91]. On the other hand, on graphs with exponential growth, it is possible that $r>0$. In many ways, the function $r(\lambda)$ is easier to study than the function $\theta(\lambda)$. The Theorem above is a result of the following proposition.

Proposition 1 For any Cayley graph $\mathcal{G}(\Lambda, \Delta)$ :

(a) The function $\lambda \mapsto r(\lambda)$ is Lipschitz continuous,

(b) $r(\lambda)>0$ implies $\theta(\lambda)>0$

(c) If $\Lambda$ is nonamenable and $\theta(\lambda)>0$, then $r(\lambda)>0$.

\section{The process seen from a typical site}

Proposition 1 (c) is proved by relating the exponential growth rate $r$ to the configuration seen from a typical infected site at a typical late time.

\section{Definitions}

The space $\mathcal{P}(\Lambda):=\{A: A \subset \Lambda\}$ of all subsets of $\Lambda$ can in a natural way be identified with $\{0,1\}^{\Lambda}$, which is a compact space under the product topology. In this topology, $\mathcal{P}_{+}(\Lambda):=\{A \in \mathcal{P}(\Lambda): A \neq \emptyset\}$ is a locally compact space. We define locally finite measures $\mu_{t}$ on $\mathcal{P}_{+}(\Lambda)$ by

$$
\mu_{t}:=\left.\sum_{i \in \Lambda} \mathbb{P}\left[\eta_{t}^{\{i\}} \in \cdot\right]\right|_{\mathcal{P}_{+}(\Lambda)} \quad(t \geq 0)
$$

where $\left.\right|_{\mathcal{P}_{+}(\Lambda)}$ denotes restriction of a measure to $\mathcal{P}_{+}(\Lambda)$. Think of $\mu_{t}$ as the law at time $t$ of the process started with one infected site, distributed according to the uniform distribution on $\Lambda$. Conditioning $\mu_{t}$ on the origin being infected yields a probability measure, describing the configuration seen from a typical infected site at time $t$.

We set

$$
\hat{\mu}_{\alpha}:=\frac{1}{Z_{\alpha}} \int_{0}^{\infty} \mu_{t} e^{-\alpha t} \mathrm{~d} t \quad(\alpha>r),
$$

where $Z_{\alpha}$ is a normalization constant such that $\hat{\mu}_{\alpha}\{A: 0 \in A\}=1$.

Proposition 2 The measures $\left\{\hat{\mu}_{\alpha}: \alpha>r\right\}$ are tight in the topology of vague convergence, and each vague limit as $\alpha \downarrow r$ yields an 'eigenmeasure' with eigenvalue $r$ (as defined below).

\section{Eigenmeasures}

By definition, we say that a locally finite measure $\mu$ on $\mathcal{P}_{+}(\Lambda)$ is an eigenmeasure with eigenvalue $\alpha$ of a contact process if

$$
\left.\int \mu(\mathrm{d} A) \mathbb{P}\left[\eta_{t}^{A} \in \cdot\right]\right|_{\mathcal{P}_{+}(\Lambda)}=e^{\alpha t} \mu \quad(t \geq 0) .
$$

Note that this says that if we start the process in the (possibly infinite) measure $\mu$, then, up to an exponential factor, we get back the same law at any later time. Consider the 'spectrum'

$$
\begin{aligned}
\mathcal{E}(\lambda):=\{\alpha \in \mathbb{R}: & \text { there exists a spatially homogeneous eigenmeasure } \\
& \text { with eigenvalue } \alpha \text { for the contact process with } \\
& \text { infection rate } \lambda\} .
\end{aligned}
$$

Proposition 2 shows that $r \in \mathcal{E}(\alpha)$. More generally, one has:

Proposition $3 \mathcal{E}(\lambda)$ is a compact subset of $\mathbb{R}$ and $r(\lambda)=$ $\max \mathcal{E}(\lambda)$.

\section{The upper invariant law}

Extending the graphical representation to negative times and setting

$$
\bar{\eta}_{t}:=\{i \in \Lambda:-\infty \rightarrow(i, t)\} \quad(t \in \mathbb{R})
$$

defines a stationary contact process $\left(\bar{\eta}_{t}\right)_{t \geq 0}$, whose stationary law $\bar{\nu}:=$ $\mathbb{P}\left[\eta_{t} \in \cdot\right]$ is called the upper invariant law. By reversing the direction of all arrows and turning the graphical representation upside down, it is not hard to see that $\bar{\nu}$ is nontrivial (i.e., concentrated on $\mathcal{P}_{+}(\Lambda)$ ) if and only if the contact process survives.

There exists a not too difficult proof, that works on any Cayley graph, that if a contact process survives, then $\bar{\nu}$ is its unique nontrivial spatially homogeneous invariant law. In a similar fashion, one can prove the following, stronger fact:

Proposition 4 If a contact process on a Cayley graph survives, then, up to a multiplicative constant, the upper invariant law $\bar{\nu}$ is the only spatially homogeneous eigenmeasure with eigenvalue zero.

\section{Proof of Proposition 1 (c) (sketch)}

Assume that a contact process on a Cayley graph survives, and its exponential growth rate $r(\lambda)$ is zero. Then, by Propositions 2 and 4 , the vague $\operatorname{limit}_{\lim } \operatorname{li0}_{\alpha} \hat{\mu}_{\alpha}$ exists and is up to a multiplicative constant equal to $\bar{\nu}$

Consider the law $\hat{\mu}_{\alpha}$, conditioned on the event $\{A: 0 \in A\}$. By our previous remarks, for $\alpha$ close to zero, this law describes a random finite set $B$, containing the origin, that looks something like this:

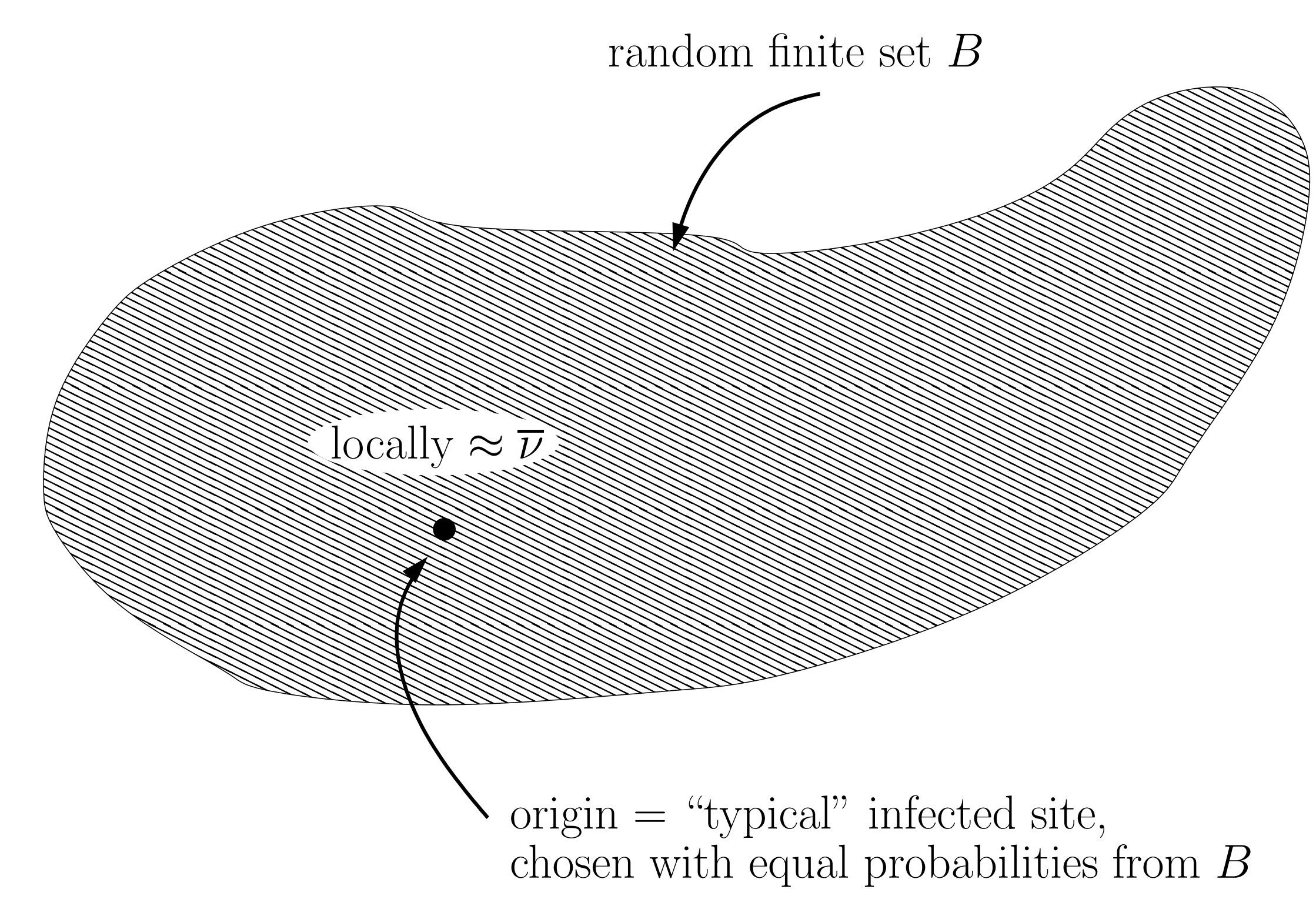

Since seen from the origin, we see something that looks like the spatially homogeneous law $\bar{\nu}$, we conclude that 0 lies with high probability far from the outer boundary of $B$. Since 0 is a 'typical' site, this contradicts nonamenability, which says that in any finite $\operatorname{set} B$, a positive fraction of the sites must lie near the boundary.

\section{References}

[BG90] C. Bezuidenhout and G. Grimmett. The critical contact process dies out. Ann. Probab. 18(4), 1462-1482, 1990

BG91] C. Bezuidenhout and G. Grimmett. Exponential decay for subcritical contact and percolation processes. Ann. Probab. 19(3), 984-1009, 1991

[BLPS99] I. Benjamini, R. Lyons, Y. Peres, and O. Schramm. Critical percolation on any nonamenable group has no infinite clusters. Ann. Probab. 27(3), 1347-1356, 1999.

[MSZ94] G.J. Morrow, R.B. Schinazi, and Y. Zhang. The critical contact process on a homogeneous tree. J. Appl. Probab. 31(1), 250-255, 1994.

[Swa08] J.M. Swart. The contact process seen from a typical infected site. Published online in J. Theor. Probab., DOI 10.1007/s10959-008-0184-4. ArXiv:math.PR/0507578. 